Research in Astronomy and Astrophysics manuscript no.

(LATEX: 51.tex; printed on September 10, 2018; 23:59)

\title{
On the diffuse soft X-ray emission from the nuclear region of M51
}

\author{
Jiren $\mathrm{Liu}^{1}$ and Shude $\mathrm{MaO}^{2,1,3}$ \\ 1 National Astronomical Observatories, Beijing 100012, China; jirenliu@bao.ac.cn \\ 2 Physics department and Tsinghua Center for Astrophysics, Tsinghua University, Beijing, 100084, China \\ 3 Jodrell Bank Centre for Astrophysics, University of Manchester, Manchester, M13 9PL, UK
}

\begin{abstract}
We present an analysis of the diffuse soft X-ray emission from the nuclear region of M51 combining both XMM-Newton RGS and Chandra data. Most of the RGS spectrum of M51 can be fitted with a thermal model with a temperature of $\sim 0.5 \mathrm{keV}$ except for the $\mathrm{O}$ VII triplet, which is forbidden-line dominated. The Fe L-shell lines peak around the southern cloud, where the $\mathrm{O}_{\mathrm{VIII}}$ and $\mathrm{N}$ VII $\mathrm{Ly} \alpha$ lines also peak. In contrast, the peak of the $\mathrm{O}_{\mathrm{VII}}$ forbidden line is about $10^{\prime \prime}$ offset from that of the other lines, indicating that it is from a spatially distinct component. The spatial distribution of the $\mathrm{O}$ VII triplet mapped by the Chandra data shows that most of the $\mathrm{O}$ viI triplet flux is located at faint regions near edges, instead of the southern cloud where other lines peak. This distribution of the $\mathrm{O}$ vII triplet is inconsistent with the photoionization model. Other mechanisms that could produce the anomalous $\mathrm{O}$ VII triplet, including a recombining plasma and charge exchange X-ray emission, are discussed.
\end{abstract}

Key words: atomic processes - plasmas - ISM: jets and outflows - galaxies: Seyfert galaxies: individual: M51 (NGC 5194) - X-rays: ISM

\section{INTRODUCTION}

The Whirlpool galaxy M51 (NGC 5194) is classified as a LINER or Seyfert 2 galaxy from studies of optical emission lines (e.g., Stauffer 1982; Ho et al. 1997). The close distance of M51 (7.8 Mpc, the mean value taken from NED1 database) allows detailed studies of its nuclear activity. Radio observations show a bipolar outflow, comprising a southern cloud and a northern loop, which is also seen in the optical emission line map (Ford et al. 1985; Crane \& van der Hulst 1992). The optical outflow with velocities as high as $1500 \mathrm{~km}$ $\mathrm{s}^{-1}$ has been reported by Cecil (1988), who also found that the arcuate radio emission of the southern cloud lies inside the bright optical emission line region, indicating a bow shock caused by the radio jet emanating from the nucleus.

The interaction between the radio jet/outflow and the surrounding gas represents an important feedback mode of active galactic nucleus (AGN), which is crucial for our understanding of galaxy evolution (e.g. Fabian 2012). X-ray observations are very effective in studying such phenomena as the radio jetdriven outflow will heat the surrounding gas to X-ray emitting temperatures. Extended X-ray emission from M51 was first detected using the Einstein Observatory (Palumbo et al. 1985), and then with ROSAT (Marston et al. 1995; Ehle et al. 1995). The BeppoSAX data of M51 showed that the X-ray emission of the nucleus is only seen directly above $10 \mathrm{keV}$, implying a neutral hydrogen column density of $N_{\mathrm{H}} \sim 10^{24}$ $\mathrm{cm}^{-2}$ (Fukazawa et al. 2001).

Observations of Einstein and ROSAT are limited by their spatial resolution. With its sub-arcsec angular resolution, Chandra has provided new insights on M51. Terashima \& Wilson (2001) found that the nuclear $\mathrm{X}$-ray morphology of M51 is similar to that seen in radio and optical observations. The X-ray spectra of the southern cloud and northern loop are similar and can be fitted by a thermal model of temperature $\sim 0.55 \mathrm{keV}$. The X-ray morphology and spectra suggest that the nuclear X-ray emitting gas of M51 are shock-heated by the bipolar radio outflow from the nucleus. Both the X-ray point sources and the extended X-ray emission from the disk of M51 have been studied using Chandra (e.g., Terashima \& Wilson 2004; Tyler et al. 2004) and XMM-Newton data (e.g., Dewangan et al. 2005; Owen \& Warwick 2009).

\footnotetext{
${ }^{1}$ http://ned.ipac.caltech.edu
} 
Table 1 List of XMM-Newton RGS observations of M51

\begin{tabular}{ccccc}
\hline ObsID & $t_{\text {tot }}(\mathrm{ks})$ & $t_{\text {eff }}(\mathrm{ks})$ & Obs time & P.A. (degrees) \\
\hline 0212480801 & 49 & 26 & $2005-07-01$ & 294 \\
0303420101 & 54 & 35 & $2006-05-20$ & 326 \\
0303420201 & 37 & 25 & $2006-05-24$ & 323 \\
0677980701 & 13 & 13 & $2011-06-07$ & 312 \\
\hline
\end{tabular}

Note: $t_{\mathrm{tot}}$ is the total exposure, $t_{\mathrm{eff}}$ is the useful exposure after removing periods of flares, and P.A. is the position angle.

All these X-ray studies are based on CCD data, for which the spectral resolution is around 10 at $1 \mathrm{keV}$. In contrast, the Reflection Grating Spectrometers (RGS) aboard the XMM-Newton telescope den Herder et al. 2001) have a better spectral resolution for moderately extended sources due to their large dispersion power. The RGS spectral resolution is $\sim 0.14 \theta \AA$, where $\theta$ (in units of arcmin) is the angular extent of the source along the dispersion direction. For M51, the spatial extent of the nuclear X-ray emitting region is about $0.5^{\prime}$, which corresponds to a spectral resolving power $\lambda / \delta_{\lambda} \sim 150$ at $15 \AA$ given the spatial resolution of XMM-Newton. With this resolution many emission lines, especially the He-like $\mathrm{O}$ viI triplet which is a diagnostic tool for the thermal state of plasma, are well resolved.

The $\mathrm{O}$ viI triplet consists of a resonance line, two inter-combination lines and a forbidden line. For an optically-thin thermal plasma in ionization equilibrium, the electron collisional excitation is efficient and favors the resonance line. In Liu et al. (2012), we studied nine nearby star-forming galaxies (including M51) and found that the forbidden lines of their O VII triplets are comparable to or even stronger than the resonance lines. We proposed the charge-exchange process between highly ionized ions and neutral species as a possible explanation. The charge-exchange captured electrons of the recipient ions are in excited states and their downward cascading favors the forbidden line (e.g. Dennerl 2010). In the special case of M51, however, the explanation is complicated by the presence of the central low-luminosity AGN and the radio jet-driven outflow.

In Liu et al. (2012), only the line ratio of the O vII triplet of M51 was studied. In this paper we provide a more detailed study of the XMM-Newton RGS spectrum of M51. In particular, we study the spatial distribution of emission lines along the cross-dispersion direction of RGS. As shown in Section3, the spatial distribution of the $\mathrm{O}$ VII triplet is different from other lines. Given this situation, the Chandra image of M51 with sub-arcsec angular resolution is helpful in revealing the $\mathrm{O}$ vII triplet distribution. Thus we also analyze the archival Chandra data of M51 with an accumulated exposure time of $700 \mathrm{ks}$, which is much deeper than the short exposure data $(\sim 15 \mathrm{ks})$ used in previous studies (e.g. Terashima \& Wilson 2001).

We describe the observational data in Section 2 and present the results of XMM-Newton and Chandra data in Section 3 and Section 4, respectively. Discussion of the results is covered in Section 5. Throughout the paper, the errors quoted are for the $90 \%$ confidence level. At a distance of $7.8 \mathrm{Mpc}, 1$ arcsec corresponds to 38 parsec.

\section{OBSERVATIONAL DATA}

The two RGSs on-board the XMM-Newton telescope are slit-less dispersive spectrometers, and photons from extended sources are recorded on CCD detectors with the dispersion angle and 1D spatial information along the cross-dispersion direction. Because the dispersion directions are different for different observations, to ensure that the dispersed spectra are from similar spatial regions, we use four archival datasets of XMM-Newton RGS observations of M51 as listed in Table 1. The maximum difference of position angles between them is about 30 degrees. The total effective exposure time is $\sim 100 \mathrm{ks}$ after removing intense flare periods.

The most recent version of the Science Analysis System (SAS 14.0) of XMM-Newton is used for the reduction of photon events. As stated above, the RGS spectrum is broadened by the spatial extent of the source along the dispersion direction. To produce a broadened redistribution matrix file (RMF), we convolve the RMF produced by the SAS tool rgsproc with a Chandra image of M51 (0.4-2 keV) using the rgsrmfsmooth tool written by Andrew Rasmussen. The broadened RMF is calculated separately for each dataset. For background subtraction, we use the model background generated by rgsproc based on the flux of CCD 9 beyond $1^{\prime}$ from the on-axis position. Because M51 lies at $\sim 1^{\prime}$ away from the on-axis position for most observations listed in Table 1, the model background is over-predicted. Indeed, the model background exceeds the observed spectrum at $\lambda>28 \AA$. Thus we scale down the model background by a factor 


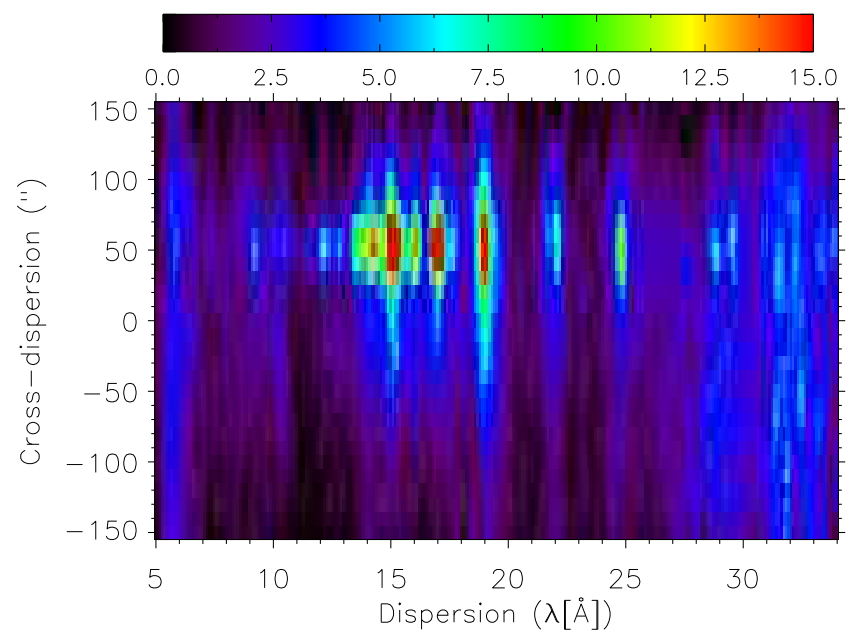

Fig. 1 Cross-dispersion vs dispersion CCD image of M51 combining both RGS1 and RGS2 data of all four observations. The emission lines are seen as vertical contours and are well resolved. The color bar is for the total event counts on linear scale. The zero point along the crossdispersion direction of ObsID 0303420201 is adopted.

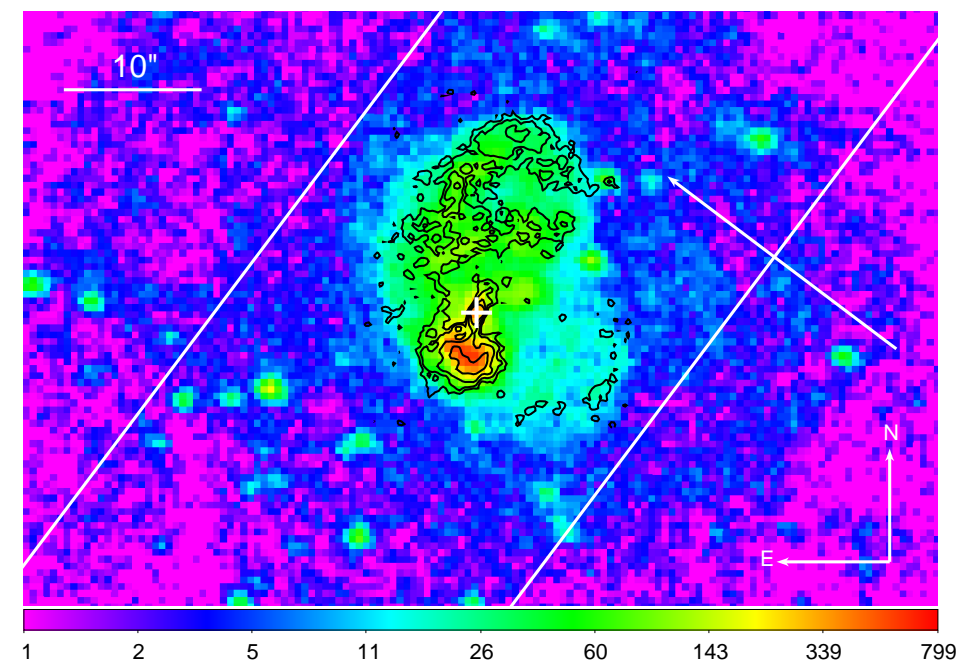

Fig. 2 Chandra counts image of M51 within $0.4-2 \mathrm{keV}$ (logarithmically scaled). The overlaid radio contours $(1.5,3,6$ and 12 in units of $10 \mu \mathrm{Jy})$ are from VLA observations at $6 \mathrm{~cm}$ wavelength by Crane \& van der Hulst (1992). The two solid lines separated by $30^{\prime \prime}$ indicate the RGS dispersion direction of ObsID 0303420201, while the arrow indicates the positive cross-dispersion direction. The white cross marks the position of the nucleus of M51, which corresponds to a cross-dispersion distance of 52" for ObsID 0303420201.

of 0.6 based on the event distribution of CCD9. Since we focus on emission lines, the uncertainty in the background subtraction will not affect our major results.

Because CCD 4 of RGS2 covering $20-24 \AA$ failed early in the mission, the data of the O vII triplet around $22 \AA$ are only from RGS1. Similarly, CCD 7 of RGS1 also failed and the data within $10.6-13.8$ $\AA$ of RGS1 are missing. Figure 1 presents the CCD image of the XMM-Newton RGS data of M51 combining both RGS1 and RGS2 for all four observations. The offsets between RGS1 and RGS2 and between different observations have been corrected. The figure shows clearly the well-resolved emission lines.

We use seven Chandra datasets with ObsID numbers of 13812, 13813, 13814, 13815, 13816, 15496, 15553, as observed by PI Kip Kuntz. After removing the flare periods, the total effective exposure time is about $700 \mathrm{ks}$. The datasets are analyzed with CIAO (version 4.6) following standard procedures. As an 


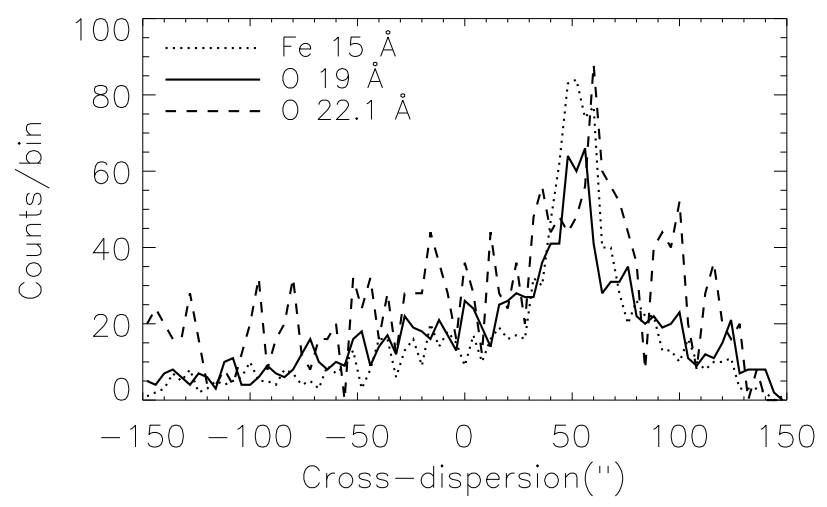

Fig. 3 Spatial distribution of the emission lines of Fe xVII at $15 \AA$, O VIII Ly $\alpha$ at $19 \AA$, and O VII forbidden line at $22.1 \AA$. For clarity, the profile of the O VII forbidden line at $22.1 \AA$ has been multiplied by a factor of 4 .

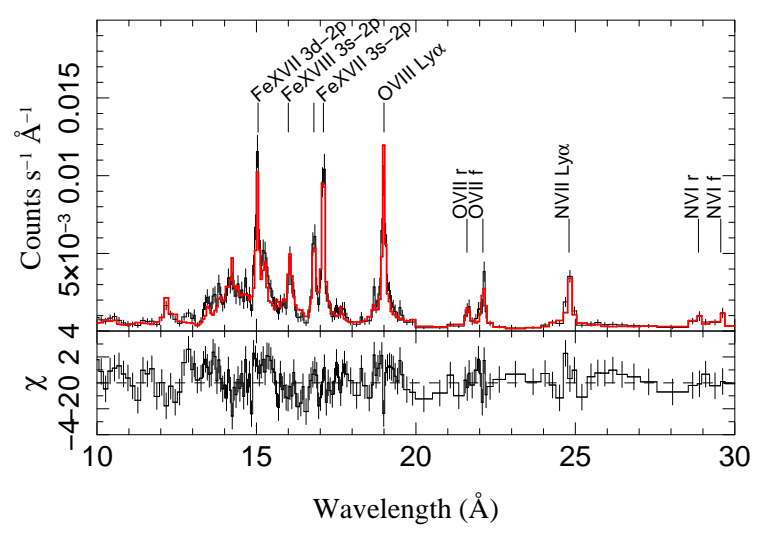

Fig. 4 Background-subtracted XMM-Newton RGS spectrum of M51 combining both RGS1 and RGS2 data of all four observations. Red histograms are the fitted thermal model with four Gaussians. $\chi$ is the difference between data and model divided by the error.

illustration, Figure 2 presents the counts image of M51 within 0.4-2 keV created by merging all the seven datasets. The over-plotted contours are from the radio $6 \mathrm{~cm}$ observation (Crane \& van der Hulst 1992), and show a close correspondence with the X-ray image. The XMM-Newton RGS dispersion direction is also illustrated in Figure 2.

\section{XMM-NEWTON RGS RESULTS}

\subsection{Spatial distribution of $\mathrm{X}$-ray emission lines}

First we study the spatial distribution of the well-resolved X-ray emission lines along the cross-dispersion direction of XMM-Newton RGS. The Fe XVII lines at 15 and $17 \AA$, the $\mathrm{O}$ VIII and $\mathrm{N}$ VII Ly $\alpha$ lines at 19 and $24.8 \AA$, and the $\mathrm{O}$ VII forbidden line at $22.1 \AA$ are studied. The wavelength regions adopted to calculate the photon counts are 14.8 - 15.5 $\AA, 16.6$ - 17.4 $\AA, 18.6$ - 19.4 $\AA, 24.4$ - $25.2 \AA$, and 21.8 - 22.4 $\AA$, respectively. We find that the profiles of Fe XVII lines at 15 and $17 \AA$ are similar, as are the $\mathrm{O}$ VIII and $\mathrm{N}$ VII Ly $\alpha$ lines. For clarity, only the profiles of Fe XVII line at $15 \AA, \mathrm{O}$ VIII Ly $\alpha$ line at $19 \AA$, and $\mathrm{O}$ VII line at $22.1 \AA$ are plotted in Figure 3. We see that the bright Fe XVII line at $15 \AA$ is centrally peaked around $50^{\prime \prime}$, with a FWHM (full width at half maximum) of $\sim 20^{\prime \prime}$. This FWHM is similar to the spatial resolution of XMM-Newton RGS. Since the cross-dispersion position of $50^{\prime \prime}$ corresponds to the location of the southern cloud, it means that the emission lines of Fe XVII are mainly from the compact southern cloud.

The $\mathrm{O}$ VIII Ly $\alpha$ line at $19 \AA$ is also peaked around $50^{\prime \prime}$, the same as the Fe xvII lines. The spatial distribution of the $\mathrm{O}$ VII forbidden line at $22.1 \AA$, however, is different from those of the other lines. Its maximum 
Table 2 Fitting results

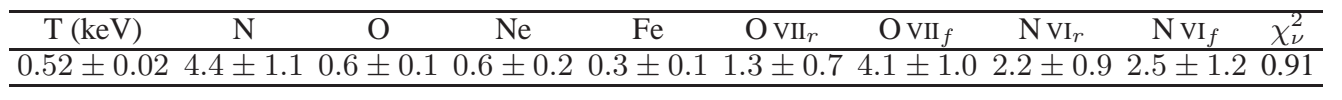

Note: the abundances are relative to the solar values; $\mathrm{OVII}_{r, f}$ and $\mathrm{NVI}_{r, f}$ (in units of $10^{-5}$ photons s $\mathrm{cm}^{-2}$ ) are the line intensities of O VII resonance line, O VII forbidden line, N VI resonance line, and N VI forbidden line, respectively.

is around $60^{\prime \prime}, 10^{\prime \prime}$ offset from the peaks of other lines. This indicates that the $\mathrm{O}$ vII forbidden line is from a spatially distinct component, compared with the other lines.

\subsection{X-ray spectrum of M51}

Since the X-ray emission lines have different spatial distributions, it is optimal to extract spectra within different spatial regions. However, the difference of the scale of $\sim 10^{\prime \prime}$ is too small to allow such spectral extractions from XMM-Newton RGS. Thus we only extract one spectrum centered on the nucleus of M51 with a cross-dispersion width about $1^{\prime}$. The background-subtracted spectrum of M51 combined from both RGS1 and RGS2 data of all four observations is plotted in Figure 4.

We see that the spectrum of M51 is dominated by emission lines. The $\mathrm{O}$ VII triplet is dominated by the forbidden line at $22.1 \AA$ and the $\mathrm{N}$ vi triplet also has a forbidden line comparable with its resonance line. The bright Fe L-shell lines around 15 - $17 \AA$ indicate that they are not from a photoionized plasma, for which the Fe L-shell lines are expected to be much weaker (e.g., Kallman et al.1996; Sako et al. 2000). They are likely due to a collisionally ionized plasma as suggested in previous studies (e.g., Terashima \& Wilson 2001). Thus we fit an optically thin collisional-ionization-equilibrium (CIE) thermal model (vapec, Foster et al. 2012) to the observed RGS spectrum of M51. Only the abundances of the elements of $\mathrm{Ne}, \mathrm{Fe}, \mathrm{O}$, and N, which show bright emission lines in the spectrum of M51, are allowed to vary. The abundances of other elements are set to solar values (Lodders 2003).

On the other hand, the forbidden-line dominated O VII triplet of M51 shows a spatial distribution different from other lines. As stated in Section 1, it is impossible to explain such an anomalous triplet by a thermal CIE model. Thus we add two Gaussians to represent the $\mathrm{O}$ vII resonance and forbidden lines. The intercombination line is too weak to be fitted. Similarly, we add another two Gaussians to represent the N vi resonance and forbidden lines. The model is subject to an absorption model of wabs (Morrison \& McCammon 1983) with a foreground neutral hydrogen column density of $2 \times 10^{20} \mathrm{~cm}^{-2}$ (Kalberla et al. 2005). The fitted results are listed in Table 2 and over-plotted in Figure 4.

The model provides a reasonable fit to the observed spectrum of M51. The fitted temperature is 0.52 $\mathrm{keV}$, similar to that obtained by Terashima \& Wilson (2001). However, the fitted abundances are higher than their values, which are only around 0.1 solar value. The low abundances they obtained are most likely due to the poor spectral resolution of Chandra data. We note that the fitted $\mathrm{O}$ and $\mathrm{N}$ abundances are not affected by the Gaussian fitting to the corresponding $\mathrm{O}$ VII and N vi triplets, since they are most likely due to a mechanism different from CIE models and at the fitted temperature, the emissivity of $\mathrm{O}$ VII and $\mathrm{N}$ vi triplets of the CIE model is weak. The residuals around the Fe and $\mathrm{O}$ lines are likely due to the simple convolution modeling of the RMF. There are residuals around 13.5 $\AA$, indicating that the Ne $\mathrm{x}$ triplet is under-estimated. The residuals around $18.6 \AA$ are likely due to the $\mathrm{O}$ VII $\mathrm{He} \beta$ line.

The temperature diagnostic $\mathrm{G}$ ratio of the He-like triplet for a thermal CIE plasma is defined as (Gabriel \& Jordan 1969)

$$
\mathrm{G}=\frac{\mathrm{f}+\mathrm{i}}{\mathrm{r}},
$$

where $\mathrm{f}, \mathrm{i}$, and $\mathrm{r}$ represents the intensity of the forbidden, inter-combination, and resonance lines, respectively. If we assume the intensity of the inter-combination line is $1 / 4$ of that of the forbidden line (Smith et al. 2001), the fitted G ratio of the O VII triplet is $3.9 \pm 2.3$. Similarly, the fitted G ratio of the $\mathrm{N}_{\text {vI }}$ triplet is $1.4 \pm 0.8$. The $\mathrm{G}$ ratio of $\mathrm{O}$ VII triplet shows that the $\mathrm{O}$ VII triplet is not due to CIE thermal plasmas, for which the $\mathrm{G}$ ratios are generally smaller than 1 . The $\mathrm{G}$ ratio of $\mathrm{N}$ vi triplet is barely consistent with a CIE model around $10^{6} \mathrm{~K}$. 


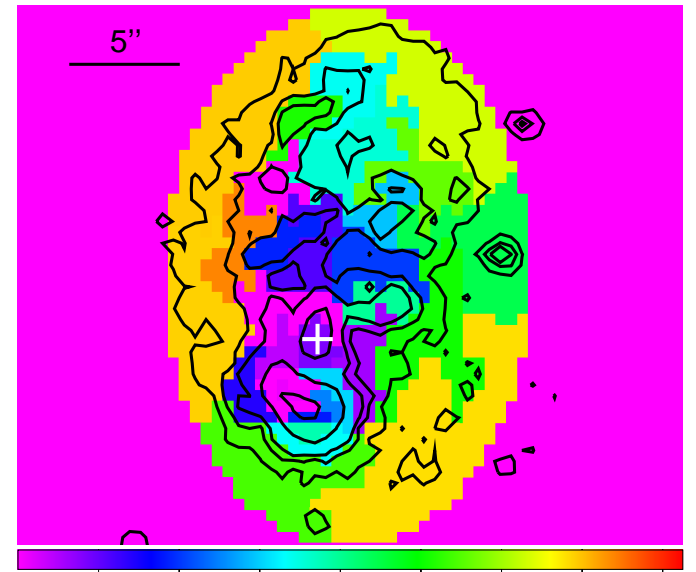

0

$4 \mathrm{E}-07$

8E-07

1.2E-06

$1.6 \mathrm{E}-06 \quad 12$

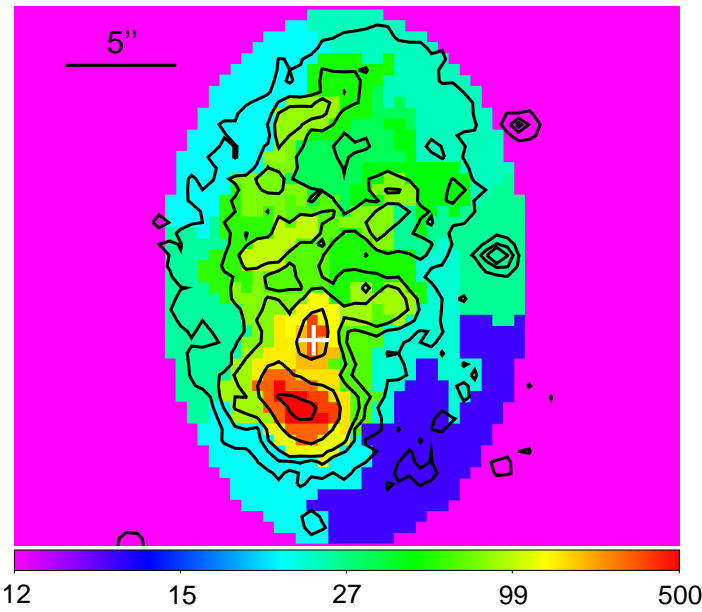

Fig. 5 Left: Fluxes of the O viI triplet obtained with the Chandra data of M51 using the contour binning method. Right: The binned counts map of M51 within $0.4-2 \mathrm{keV}$. The contours of levels of 20,50,80, 200, and 550 for unbinned map are plotted in both panels. The white cross marks the nucleus of M51.

\section{Chandra RESULTS}

As shown in Section3.1, the peak of the spatial profile of the $\mathrm{O}$ vil forbidden line is offset from those of the other lines by $\sim 10^{\prime \prime}$. This scale can be easily resolved with Chandra data. Thus we analyze the Chandra data of M51 in this section.

We use the contour binning method (Sanders 2006) to divide the nuclear X-ray emitting region of M51 into 34 different bins with a minimum signal to noise ratio of 40 . The X-ray spectrum is extracted from each bin with a background spectrum taken from a source-free region outside the nuclear region. We fit a thermal CIE model with variable $\mathrm{O}, \mathrm{Ne}, \mathrm{Mg}$, and $\mathrm{Fe}$ abundances and limit the fitting range to $0.4-1.5$ $\mathrm{keV}$. We include a Gaussian (with a center of $21.85 \AA$ and a width of $0.25 \AA$ ) to represent the $\mathrm{O}$ vil triplet. Adding a Gaussian will improve the $\chi^{2}$ by 4.3 on average. The improvement of $\chi^{2}$ is 8.1 for the spectra with the Gaussian line fluxes larger than $5 \times 10^{-7}$ photons $\mathrm{s}^{-1} \mathrm{~cm}^{-2}$. We also tested the fitting method by fitting mock spectra with a line flux of $1 \times 10^{-6}$ photons s $\mathrm{s}^{-1} \mathrm{~cm}^{-2}$, and found that about $75 \%$ of the fitted fluxes are within 3 sigma of the true value.

Due to the limited spectral resolution of Chandra data, we do not expect the fitted O vil triplet fluxes to be as accurate as the measurement by the XMM-Newton RGS data. Nevertheless, it is likely to provide a qualitative distribution of the $\mathrm{O}$ vII triplet, especially for bins with high $\mathrm{O}$ vII triplet fluxes. The fitted fluxes of the $\mathrm{O}$ VII triplet from the Chandra data are plotted in the left panel of Figure 5. For comparison, the binned counts image is plotted in the right panel. We see that the fluxes of the $\mathrm{O}$ VII triplet are generally higher for faint bins near the edges. It shows that the fitted $\mathrm{O}$ viI triplet has a more extended distribution than that of the total emission within $0.4-2 \mathrm{keV}$, which is centered on the southern cloud. The northern regions have more $\mathrm{O}$ VII triplet flux than the southern part. This is consistent with the XMM-Newton result. Another interesting feature to note is that the southern cloud seems to be enclosed by an arc of O vII triplet in the outward direction.

\section{DISCUSSION}

We have analyzed the XMM-Newton RGS spectrum of M51, the emission lines of which are well resolved. Most of the spectrum of M51 can be fitted with a thermal CIE model of temperature $\sim 0.5 \mathrm{keV}$, except for the $\mathrm{O}$ VII triplet, which is forbidden-line dominated. The Fe xVII lines at 15 and $17 \AA$ are centrally peaked around the cross-dispersion position of $50^{\prime \prime}$ with a FWHM of $20^{\prime \prime}$. This indicates that they are mainly from the compact southern cloud. The $\mathrm{O}$ VIII Ly $\alpha$ line at $19 \AA$ and $\mathrm{N}$ VII Ly $\alpha$ line at $25 \AA$ also peak around the same position as the Fe xVII lines. In contrast, the peak of the $\mathrm{O}$ VII forbidden line is about $10^{\prime \prime}$ offset from the other lines. The O VII triplet map obtained with the Chandra data shows that most of the fluxes of the $\mathrm{O}$ VII triplet are located at faint regions, instead of the southern cloud where the other lines peak around. 
As stated in Section 1, the forbidden-line-dominated O VII triplet of M51 cannot be due to a thermal CIE plasma, and we have proposed the charge-exchange process as a possible explanation. Nevertheless, the existence of low-luminosity AGN and the radio jet-driven outflow in the nuclear region of M51 makes other explanations possible, including photoionization and non-equilibrium-ionization plasmas. Below we discuss them in turn.

For a photoionized plasma, the emission is dominated by recombination, which also favors the forbidden line. One characteristic feature of the photoionization model is the spatial profile. As the central AGN is the ionizing source, the ionizing flux will decline as $r^{-2}$ and photoionization is most important for regions close to AGN. This has been illustrated by the optical study of M51 (Bradley et al. 2004), which shows that photoionization is dominant within the inner region $\left(r<1^{\prime \prime}\right)$ and the shock model is preferred outside $\left(r \sim 2.5^{\prime \prime}\right)$. The mapped morphology of the O vil triplet from Chandra data shows no fluxes around the nucleus and is most prominent at the outer regions. This is inconsistent with the photoionization model.

All the optical, radio and X-ray studies of M51 support a scenario in which the nuclear gas of M51 is shock-heated by the radio outflow emanating from the nucleus Cecil 1988; Ford et al. 1985; Terashima \& Wilson 2001). In this situation, the non-equilibrium-ionization (NEI) plasma is another possible explanation for the anomalous $\mathrm{O}$ VII triplet. If a plasma is shock-heated suddenly, the ionization process lags behind heating, and will result in an ionizing plasma. The inner-shell collisional ionization of $\mathrm{O}^{5+}$ ions can lead to excited $\mathrm{O}^{6+}$ ions and enhance the forbidden line emission (e.g., Liedahl 1999). However, this only happens in a short transition period when the $\mathrm{O}^{5+}$ fraction is non-negligible. Using the Sedov model (Borkowski et al. 2001), we find that the $\mathrm{G}$ ratio of the $\mathrm{O}$ vII triplet is around 3 at an ionization time $n_{e} t \sim 3.5 \times 10^{9} \mathrm{~cm}^{-3} \mathrm{~s}$. This timescale is too short compared with the spatial extent we studied. A more likely explanation is a recombining NEI plasma.

When a blast wave expands into a rarefied medium, a recombining plasma could be produced due to rapid adiabatic cooling, as proposed to explain the radiative recombination continuum features in some supernova remnants (SNRs) (e.g., Yamaguchi et al. 2012). The spatial distribution of the O vil triplet mapped by the Chandra data is consistent with this scenario. A unique test of a recombining plasma is the associated radiative recombination continuum. However, due to the presence of strong Fe lines, it is hard to tell whether there is a radiative recombination continuum of O VII (at 16.8 $\AA$ ) in the spectrum of M51.

On the other hand, to explain the anomalous $\mathrm{O}$ vil triplet with the charge-exchange process, the interacting interfaces between the highly ionized gas and neutral species are needed. The observed HI (Walter et al. 2008) and CO (Koda et al. 2011) maps of M51 are well correlated with the spirals. CO is also detected on 1-2 arcsec scales around the nucleus (Matsushita et al. 2007). Currently there is no much information of neutral materials on $10^{\prime \prime}$ scales of M51. Further observations are needed to test the existence of neutral materials on $10^{\prime \prime}$ scale. Different from the recombining plasma, the charge-exchange model shows no radiative recombination continuum, but has enhanced emission lines from preferred energy levels $(n=4-7$ for OVII) (e.g. Beiersdorfer et al. 2003). Future spatially-resolved high-resolution X-ray spectroscopy by the soft X-ray spectrometer of Astro-H will be able to test the existence of the radiative recombination continuum of M51.

Acknowledgements We thank the referee for their valuable comments and Lijun Gou and Richard Long for reading of the draft. This work is supported by a National Natural Science Foundation of China for Young Scholar Grant (11203032), and by the Strategic Priority Research Program "The Emergence of Cosmological Structures" of the Chinese Academy of Sciences Grant No. XDB09000000 and NSFC grant 11333003 (SM). This research has made use of XMM-Newton archival data. XMM-Newton is an ESA science mission with instruments and contributions directly funded by ESA Member States and the USA (NASA). In addition, the research used observations obtained with the Chandra X-ray observatory, which is operated by Smithsonian Astronomical Observatory on behalf of NASA.

\section{References}

Beiersdorfer, P., Boyce, K. R., Brown, G. V., Chen, H., et al. 2003, Science, 300, 1558

Bradley, L. D., Kaiser, M. E., Baan, W. A. 2004, ApJ, 603, 463

Borkowski, K. J., Lyerly, W. J., Reynolds, S. P. 2001, ApJ, 548, 820

Cecil, G. 1988, ApJ, 329, 38

Crane, P. C. \& van der Hulst, J. M. 1992, AJ, 103, 1146

den Herder, J. W. et al. 2001, A\&A, 365, L7

Dennerl, K. 2010, SSR., 157, 57 
Dewangan, G. C., Griffiths, R. E., Choudhury, M., Miyaji, T., Schurch, N. J. 2005, ApJ, 635, 198

Ehle, M., Pietsch, W., Beck, R. 1995, A\&A, 295, 289

Fabian, A. C. 2012, ARA\&A, 50, 455

Ford, H. C., Crane, P. C., Jacoby, G. H., Lawrie, D. G., van der Hulst, J. M. 1985, ApJ, 293, 132

Foster, A. R., Ji, L., Smith, R. K. and Brickhouse, N. S. 2012, ApJ, 756, 128

Fukazawa, Y., Iyomoto, N., Kubota, A., Matsumoto, Y., Makishima, K. 2001, A\&A, 374, 73

Gabriel, A. H. \& Jordan, C. 1969, MNRAS, 145, 241

Ho, L. C., Filippenko, A. V., Sargent, W. L. W. 1997, ApJS, 112, 315

Kalberla, P. M. W., Burton, W. B., Hartmann, D., Arnal, E. M., Bajaja, E., Morras, R., Poppel, W. G. L. Astronomy \& Astrophysics, 440, 775

Kallman, T. R., Liedahl, D., Osterheld, A., Goldstein, W., Kahn, S. 1996, ApJ, 465, 994

Kinkhabwala, A. et al. 2002, ApJ, 575, 732

Koda, J., Sawada, T., Wright, M. C. H., Teuben, P., Corder, S. A., Patience, J., Scoville, N., Donovan Meyer, J., Egusa, F. 2011, ApJS, 193, 19

Liedahl, D. A. 1999, X-Ray Spectroscopy in Astrophysics, edited by J. V. Paradijs and J. A. M. Bleeker, Lecture Notes in Physics, 520, 189

Liu, J., Wang, Q. D., Mao, S. 2012, MNRAS, 420, 3389

Lodders, K. 2003, ApJ, 591, 1220

Marston, A. P., Elmegreen, D., Elmegreen, B., Forman, W., Jones, C., Flanagan, K. 1995, ApJ, 438, 663

Matsushita, S., Muller, S., Lim, J. 2007, A\&A, 468, L49

Morrison, R. \& McCammon, D. 1983, ApJ, 270, 119

Owen, R. A. \& Warwick, R. S. 2009, MNRAS, 394, 17410

Palumbo, G. G. C., Fabbiano, G., Trinchieri, G., Fransson, C. 1985, ApJ, 298, 259

Sako, M., Kahn, S. M., Paerels, F., Liedahl, D. A. 2000, ApJ, 543, L115

Sanders, J. S. 2006, MNRAS, 371, 829

Smith, R. K., Brickhouse, N. S., Liedahl, D. A., \& Raymond, J. C. 2001, ApJL, 556, L91

Stauffer, J. R. 1982, ApJS, 50, 517

Terashima, Y. \& Wilson, A. S. 2001, ApJ, 560, 139

Terashima, Y. \& Wilson, A. S. 2004, ApJ, 601, 735

Tyler, K., Quillen, A. C., LaPage, A., Rieke, G. H. 2004, ApJ, 610, 213

Walter, F., Brinks, E., de Blok, W. J. G., Bigiel, F., Kennicutt, R. C., Jr., Thornley, M. D., Leroy, A. 2008, AJ, 136, 2563

Yamaguchi, H., Ozawa, M., Ohnishi, T. 2012, AdSpR, 49, 451 